\title{
On the shape of solutions of an asymptotically linear problem
}

\author{
MASSIMO GROSSI
}

Abstract. In this paper we study the problem

$$
\begin{cases}-\Delta u=|u|^{\epsilon} u & \text { in } \Omega \\ u=0 & \text { on } \partial \Omega\end{cases}
$$

where $\Omega$ is a smooth bounded domain of $\mathbb{R}^{N}, N \geq 1, \epsilon>0$. We will show that, under some assumptions, the solutions to (0.1) are close to suitable linear combinations of eigenfunctions of the problem

$$
\begin{cases}-\Delta u=\lambda u & \text { in } \Omega \\ u=0 & \text { on } \partial \Omega .\end{cases}
$$

Mathematics Subject Classification (2000): 35J60.

\section{Introduction and statement of the main results}

Let us consider the following problem

$$
\begin{cases}-\Delta u=|u|^{p-1} u & \text { in } \Omega \\ u=0 & \text { on } \partial \Omega\end{cases}
$$

where $p>1$ and $\Omega$ is a smooth bounded domain of $\mathbb{R}^{N}, N \geq 1$. Problem (1.1) has been extensively studied in the last years; there is a wide literature on existence, multiplicity and qualitative properties of solutions to (1.1) as $p$ and $\Omega$ vary. Let us recall a classical result (see [7] for a proof).

Theorem 1.1. Let $1<p<\frac{N+2}{N-2}$. Then there exist infinitely many pairs of solution to (1.1).

In this paper we study (1.1) when $p$ is close to 1 , i.e. $p=1+\epsilon$ with $\epsilon$ positive small enough and we try to characterize its set of solutions. Setting $p=1+\epsilon(1.1)$ becomes

$$
\begin{cases}-\Delta u=|u|^{\epsilon} u & \text { in } \Omega \\ u=0 & \text { on } \partial \Omega .\end{cases}
$$

Supported by M.I.U.R., project "Variational methods and nonlinear differential equations". Received May 29, 2008; accepted in revised form October 23, 2008. 
To our knowledge in this case there are not many results. One of them is due to C. S. Lin (see [5]):

Theorem 1.2. Let $\Omega$ be a convex domain and $p$ sufficiently close to 1 . Then the positive solution to (1.2) is unique.

In [5] a crucial tool is the scaling invariance of the problem. Theorem 1.2 was extended by Dancer (see [3]) without assuming the convexity of the domain. Another result for a similar problem can be found in [4].

In this paper we will use a different approach and we study the set of solutions to (1.2) as $\epsilon \rightarrow 0$. Roughly speaking, we show that, for $\epsilon$ small enough, the problem (1.2) is "close" to the eigenvalue problem

$$
\begin{cases}-\Delta u=\lambda u & \text { in } \Omega \\ u=0 & \text { on } \partial \Omega\end{cases}
$$

Let us denote by $\sigma(-\Delta)$ the set of the eigenvalues of $-\Delta$ with Dirichlet boundary condition and, for any $\lambda \in \sigma(-\Delta)$, by $m(\lambda)$ the multiplicity of the eigenvalue $\lambda$. Finally, for any $\lambda \in \sigma(-\Delta)$, let $W^{(\lambda)}=\operatorname{span}\left\{e_{1}^{(\lambda)}, . ., e_{m(\lambda)}^{(\lambda)}\right\}$ be the eigenspace associated to the eigenvalue $\lambda$ with $\int_{\Omega} e_{i}^{(\lambda)} e_{j}^{(\lambda)}=\delta_{i}^{j}$.

We have the following result:

Theorem 1.3. Let $\bar{\lambda} \in \sigma(-\Delta)$. Then there exists $\epsilon_{0}=\epsilon_{0}(\bar{\lambda}, N, \Omega)>0$ such that for any $0<\epsilon<\epsilon_{0}$ and for any $\lambda \leq \bar{\lambda}, \lambda \in \sigma(-\Delta)$, we have that (1.2) has the solutions

$$
u_{j, \epsilon}= \pm \lambda^{\frac{1}{\epsilon}}\left(\sum_{i=1}^{m(\lambda)} \gamma_{i}^{(j, \lambda)} e_{i}^{(\lambda)}+\phi_{\epsilon}\right) \quad j=1, . ., m(\lambda)
$$

where $\phi_{\epsilon} \rightarrow 0$ in $C^{2}(\Omega)$ and $\left(\gamma_{1}^{(j, \lambda)}, . ., \gamma_{m(\lambda)}^{(j, \lambda)}\right)$ is a critical point of the functional $J: \mathbb{R}^{m(\lambda)} \rightarrow \mathbb{R}$

$$
J\left(\Gamma_{1}, . ., \Gamma_{m(\lambda)}\right)=\int_{\Omega}\left|\left(\sum_{i=1}^{m(\lambda)} \Gamma_{i} e_{i}^{(\lambda)}\right)\right|^{2}\left(2 \log \left|\sum_{i=1}^{m(\lambda)} \Gamma_{i} e_{i}^{(\lambda)}\right|-1\right)
$$

for any $j=1, . ., m(\lambda)$.

Hence we have that any eigenspace $W^{(\lambda)}$ associated to $\lambda$ generates at least $m(\lambda)$ solutions to (1.2) provided that $\epsilon$ is small enough. The proof of Theorem 1.3 uses a finite dimensional reduction of Liapounov-Schmidt type, a tool widely used in perturbation problem. Note that we do not require any "non-degeneracy" assumption on the critical points $\gamma_{1}^{(1, \lambda)}, \ldots, \gamma_{1}^{(m(\lambda), \lambda)}$.

Our aim is now to show that, under an a priori estimate on the solutions, the result in Theorem 1.2 is sharp. We have the following: 
Theorem 1.4. Let $u_{n}$ be a sequence of nontrivial solutions to

$$
\begin{cases}-\Delta u=|u|^{\epsilon_{n}} u & \text { in } \Omega \\ u=0 & \text { on } \partial \Omega\end{cases}
$$

with $\epsilon_{n} \rightarrow 0$ as $n \rightarrow+\infty$ and suppose that there exist $M \geq \lambda_{1}\left(\lambda_{1}\right.$ is the first eigenvalue in $\sigma(-\Delta)$ ) and $C>0$ such that

$$
\int_{\Omega}\left|u_{n}\right|^{2} \leq C M^{\frac{2}{\epsilon_{n}}}
$$

Then there exists $\lambda \in \sigma(-\Delta)$ such that, up to a subsequence again denoted by $u_{n}$,

$$
\left(\int_{\Omega}\left|u_{n}\right|^{2}\right)^{\epsilon_{n}} \rightarrow \lambda \in \sigma(-\Delta), \quad \text { as } n \rightarrow+\infty,
$$

and

$$
u_{n}=\lambda^{\frac{1}{\epsilon_{n}}}\left(\sum_{i=1}^{m(\lambda)} \gamma_{i} e_{i}^{(\lambda)}+\phi_{n}\right)
$$

with $\phi_{n} \rightarrow 0$ in $C^{2}(\Omega)$ as $n \rightarrow+\infty$ and $\left(\gamma_{1}, \ldots, \gamma_{m}(\lambda)\right)$ is a critical point of the functional (1.4).

At this stage a question arises: Does each $W^{(\lambda)}$ generate exactly $m(\lambda)$ solutions? The answer is affirmative under a non-degeneracy assumption on the critical points of the functional (1.4).

Theorem 1.5. Let us suppose that for any $\lambda \leq \bar{\lambda}$ the function $J$ defined in (1.4) has exactly $m(\lambda)$ nondegenerate critical points $\gamma^{(1)}, \ldots, \gamma^{(m(\lambda))} \in \mathbb{R}^{m(\lambda)}$. Then, for $\epsilon$ small enough, (1.2) admits exactly $\sum_{i=1}^{\bar{\lambda}} m\left(\lambda_{i}\right)$ pairs of nontrivial solutions satisfying

$$
\int_{\Omega}\left|u_{\epsilon}\right|^{2} \leq C M^{\frac{2}{\epsilon}}
$$

for some $M \geq \lambda_{1}$ and $C>0$.

Note that if the eigenvalue is simple (i.e. $m(\lambda)=1)$ it is possible to prove that any critical point of the functional $J$ is nondegenerate (see proof of the Corollary 1.6). Then we have the following:

Corollary 1.6. Suppose that the any eigenvalue in $\sigma(-\Delta)$ is simple. Then for any $\lambda_{k} \in \sigma(-\Delta)$ there exists $\bar{\epsilon}_{0}=\bar{\epsilon}_{0}(\bar{\lambda}, N, \Omega)>0$ such that for any $0<\epsilon<\epsilon_{0}(1.2)$ has exactly $k$ pairs of nontrivial solutions satisfying (1.9).

The last corollary can be applied to many situations; indeed, from a Micheletti's result (see [6]) we have that all eigenvalues of $-\Delta$ are simple for "generic" domains close to $\Omega$ (see also [8]). 
After this result was completed we have learned that some ideas of the paper can be found in [1] where the authors proved some qualitative properties of the solutions to (1.1).

The paper is organized as follows: in Sections 2-3 we give the proof of Theorem 1.3. In Section 4 we prove Theorem 1.4 and in Section 5 we prove Theorem 1.5 and its corollary.

\section{The Lyapunov-Schmidt reduction}

Let us denote by $\sigma(-\Delta)$ the set of the eigenvalues of $-\Delta$ with zero Dirichlet boundary condition and, for any $\lambda \in \sigma(-\Delta), m(\lambda)$ be the multiplicity of the eigenvalue $\lambda$. Finally, for any $\lambda \in \sigma(-\Delta)$, denote by $W_{k}^{(\lambda)}=\operatorname{span}\left\{e_{1}^{(\lambda)}, . ., e_{m(\lambda)}^{(\lambda)}\right\}$ the eigenspace associated to the eigenvalue $\lambda$.

In this section we fix $\lambda \in \sigma(-\Delta)$ and set $k=m(\lambda), e_{i}^{(\lambda)}=e_{i}$, for $i=$ $1, . ., m(\lambda)$ and $W_{k}^{(\lambda)}=W_{k}$. For $\gamma=\left(\gamma_{1}, . ., \gamma_{k}\right) \in \mathbb{R}^{k}$ we look for solutions to (1.2) as

$$
u_{\epsilon}(x)=\lambda^{\frac{1}{\epsilon}}\left(\sum_{i=1}^{k} \gamma_{i} e_{i}(x)+\phi(x)\right) \quad \text { with } \phi \in H^{2}(\Omega) \cap H_{0}^{1}(\Omega)
$$

where $H^{2}(\Omega)$ and $H_{0}^{1}(\Omega)$ denote the usual Sobolev spaces.

Set by $\|u\|=\|u\|_{H^{2}(\Omega) \cap H_{0}^{1}(\Omega)}$ and $\|u\|_{2}=\|u\|_{L^{2}(\Omega)}$. Moreover we require that

$$
|\gamma| \leq \gamma_{0}
$$

where $\gamma_{0}$ will be chosen later.

Set

$$
W_{k}^{\perp}=\left\{u \in H^{2}(\Omega) \cap H_{0}^{1}(\Omega): \int_{\Omega} u e_{i}=0 \text { for any } i=1, . ., k\right\} .
$$

Our first step is to derive the equation satisfied by the function $\phi$. Inserting (2.1) in (1.2) we get

$$
-\Delta\left(\sum_{i=1}^{k} \gamma_{i} e_{i}+\phi\right)=\lambda\left|\left(\sum_{i=1}^{k} \gamma_{i} e_{i}+\phi\right)\right|^{\epsilon}\left(\sum_{i=1}^{k} \gamma_{i} e_{i}+\phi\right),
$$

and using the mean value theorem we get that $\phi$ verifies

$$
-\Delta \phi-\lambda \phi=A(\phi)
$$

with $A: H^{2}(\Omega) \rightarrow L^{2}(\Omega)$,

$$
A(\phi)=\lambda\left|\sum_{i=1}^{k} \gamma_{i} e_{i}+\phi\right|^{\epsilon}\left(\sum_{i=1}^{k} \gamma_{i} e_{i}+\phi\right)-\lambda\left(\sum_{i=1}^{k} \gamma_{i} e_{i}+\phi\right) .
$$


Since the operator $-\Delta-\lambda I$ has a nontrivial kernel spanned by $\left\{e_{1}, \ldots, e_{k}\right\}$ we will solve equation (2.5) in $W_{k}^{\perp}$. To this end let us denote by $\Pi_{k}: H^{2}(\Omega) \cap H_{0}^{1}(\Omega) \rightarrow$ $W_{k}$ and $\Pi_{k}^{\perp}: H^{2}(\Omega) \cap H_{0}^{1}(\Omega) \rightarrow W_{k}^{\perp}$ the projections on $W_{k}$ and $W_{k}^{\perp}$ respectively. So we try to solve the problem

$$
\Pi_{k}^{\perp}[-\Delta \phi-A(\phi)]=0 .
$$

Let us introduce $\mathcal{L}: W_{k}^{\perp} \rightarrow W_{k}^{\perp}$ defined by

$$
\mathcal{L}=\Pi_{k}^{\perp}(-\Delta-\lambda I)
$$

By classical results we get that

$$
\|\mathcal{L}\| \geq C
$$

In this setting (2.7) is equivalent to find a function $\phi \in W_{k}^{\perp}$ which verifies

$$
\phi=\mathcal{L}^{-1}\left[\Pi_{k}^{\perp}(A(\phi))\right]
$$

Proposition 2.1. Let $\gamma \neq 0, \gamma \leq \gamma_{0}$. Then there exists $\epsilon_{0}>0$ such that for any $0<\epsilon<\epsilon_{0}$ there exists a unique $\phi_{\epsilon} \in W_{k}^{\perp}, \phi_{\epsilon}=\phi_{\epsilon}(\gamma, x)$ which verifies (2.10). Moreover $\left\|\phi_{\epsilon}\right\| \leq \delta \epsilon$ with $\delta$ depending only on $\lambda, \gamma_{0}, N, \Omega$.

Proof. By the mean value theorem we can write $A$ in the following way,

$$
A(\phi)=\epsilon \lambda\left(\sum_{i=1}^{k} \gamma_{i} e_{i}+\phi\right) \log \left|\sum_{i=1}^{k} \gamma_{i} e_{i}+\phi\right| \int_{0}^{1}\left|\sum_{i=1}^{k} \gamma_{i} e_{i}+\phi\right|^{t \epsilon} d t
$$

Since $\phi$ is bounded in $H^{2}(\Omega)$ it is not difficult to show that $A(\phi) \in L^{2}(\Omega)$. Let us introduce the operator $F: W_{k}^{\perp} \rightarrow W_{k}^{\perp}$

$$
F(\phi)=\mathcal{L}^{-1}\left[\Pi_{k}^{\perp}(A(\phi))\right]
$$

and let us show that $F$ is a contraction from $B_{\epsilon}=\left\{\phi \in W_{k}^{\perp}:\|\phi\| \leq \delta \epsilon\right\}$ into itself ( $\delta$ will be chosen later).

We divide the proof in two steps. 
Step 1: $F$ maps $B_{\epsilon}$ into itselffor a suitable $\delta$ and $\epsilon$ small enough.

We have that

$$
\begin{aligned}
& \int_{\Omega}|A(\phi)|^{2} \\
& =\lambda^{2} \epsilon^{2} \int_{\Omega}\left(\log \left|\sum_{i=1}^{k} \gamma_{i} e_{i}+\phi\right|\left(\sum_{i=1}^{k} \gamma_{i} e_{i}+\phi\right) \int_{0}^{1}\left|\sum_{i=1}^{k} \gamma_{i} e_{i}+\phi\right|^{t \epsilon} d t\right)^{2} \\
& \leq \lambda^{2} \epsilon^{2} \int_{\Omega}\left(\left.\int_{0}^{1} \log \left|\sum_{i=1}^{k} \gamma_{i} e_{i}+\phi\right|\left|\sum_{i=1}^{k} \gamma_{i} e_{i}+\phi\right|\right|^{1+t \epsilon} d t\right)^{2} \\
& \leq\left(\text { using that }|\log | t|\| x|^{1+t \epsilon} \leq C\left(|t|^{\frac{1}{2}}+|t|^{\frac{q}{2}}\right) \text { for some } 2<q \leq \frac{2 N}{N-2}\right) \\
& C \epsilon^{2} \int_{\Omega}\left(\left|\sum_{i=1}^{k} \gamma_{i} e_{i}+\phi\right|+\left|\sum_{i=1}^{k} \gamma_{i} e_{i}+\phi\right|^{q}\right) \leq C \epsilon^{2}\left(1+\int_{\Omega}|\phi|+\int_{\Omega}|\phi|^{q}\right) \\
& \leq C \epsilon^{2}\left(1+\|\phi\|+\|\phi\|^{q}\right) .
\end{aligned}
$$

Then we have that

$$
\|A(\phi)\|_{2} \leq C \epsilon\left(1+\|\phi\|^{\frac{1}{2}}+\|\phi\|^{\frac{q}{2}}\right)
$$

and from the definition of $F$ we get

$$
\|F(\phi)\| \leq\|\mathcal{L}\|\left\|\Pi_{k}^{\perp}\right\|\|A(\phi)\|_{2} \leq C_{0} \epsilon\left(1+\|\phi\|^{\frac{1}{2}}+\|\phi\|^{\frac{q}{2}}\right) .
$$

Assuming that $\|\phi\| \leq \delta \epsilon$ with $\delta=2 C_{0}$ by (2.16) we get

$$
\|F(\phi)\| \leq 2 C_{0} \epsilon
$$

which gives the claim of this step.

Step 2: $F$ is a contraction mapping from $B_{\epsilon}$ into itself for $\epsilon$ small enough. 
Let us fix $\delta=2 C_{0}$ as in the previous step. We have

$$
\begin{gathered}
\left\|F\left(\phi_{1}\right)-F\left(\phi_{2}\right)\right\| \leq\|\mathcal{L}\|\left\|\Pi_{k}^{\perp}\right\|\left\|A\left(\phi_{1}\right)-A\left(\phi_{2}\right)\right\|_{2} \\
\leq C \| \sum_{i=1}^{k} \gamma_{i} e_{i}+\left.\phi_{1}\right|^{\epsilon}\left(\sum_{i=1}^{k} \gamma_{i} e_{i}+\phi_{1}\right)-\left(\phi_{1}-\phi_{2}\right) \\
-\left|\sum_{i=1}^{k} \gamma_{i} e_{i}+\phi_{2}\right|^{\epsilon}\left(\sum_{i=1}^{k} \gamma_{i} e_{i}+\phi_{2}\right) \|_{2}
\end{gathered}
$$

(using the mean value theorem)

$$
=C\left\|\int_{0}^{1}\left[(1+\epsilon)\left|\sum_{i=1}^{k} \gamma_{i} e_{i}+s \phi_{1}+(1-s) \phi_{2}\right|^{\epsilon}-1\right] d s\left(\phi_{1}-\phi_{2}\right)\right\|_{2} \text {. }
$$

Let us split $\Omega$ in the following way,

$$
\Omega=D_{1, \epsilon, s} \cup D_{2, \epsilon, s} \cup D_{3, \epsilon, s}
$$

where

$$
\begin{aligned}
& D_{1, \epsilon, s}=\left\{x \in \Omega:\left|\sum_{i=1}^{k} \gamma_{i} e_{i}+s \phi_{1}+(1-s) \phi_{2}\right| \leq \epsilon\right\}, \\
& D_{2, \epsilon, s}=\left\{x \in \Omega: \epsilon<\left|\sum_{i=1}^{k} \gamma_{i} e_{i}+s \phi_{1}+(1-s) \phi_{2}\right|<2\left\|\sum_{i=1}^{k} \gamma_{i} e_{i}\right\| \|_{\infty}\right\}, \\
& D_{3, \epsilon, s}=\left\{x \in \Omega:\left|\sum_{i=1}^{k} \gamma_{i} e_{i}+s \phi_{1}+(1-s) \phi_{2}\right| \geq 2\left\|\sum_{i=1}^{k} \gamma_{i} e_{i}\right\|_{\infty}\right\},
\end{aligned}
$$

and also split the integral in (2.18) as

$$
\begin{aligned}
& \left\|\int_{0}^{1}\left[\left|\sum_{i=1}^{k} \gamma_{i} e_{i}+s \phi_{1}+(1-s) \phi_{2}\right|^{\epsilon}-1\right] d s\left(\phi_{1}-\phi_{2}\right)\right\|_{2}^{2} \\
& =\int_{D_{1, \epsilon, s}} \cdot+\int_{D_{2, \epsilon, s}} \cdot+\int_{D_{3, \epsilon, s}} \cdot=I_{1}+I_{2}+I_{3} .
\end{aligned}
$$

Estimate of $I_{1}$

First let us estimate the measure of $D_{1, \epsilon, s}$. We want to show that

$$
\operatorname{meas}\left(D_{1, \epsilon, s}\right) \rightarrow 0 \quad \text { as } \epsilon \rightarrow 0 \forall s \in[0,1] \text {. }
$$

By contradiction let us suppose that there exists $\epsilon_{0}>0, \bar{s} \in[0,1]$ such that

$$
\operatorname{meas}\left(D_{1, \epsilon, \bar{s}}\right) \geq \delta>0 \quad \forall \epsilon \in\left(0, \epsilon_{0}\right) \text {. }
$$


Set

$$
D=\bigcap_{n \geq 1} D_{1, \frac{1}{n}, \bar{s}}
$$

Of course we have that

$$
\operatorname{meas}(D) \geq \delta
$$

and

$$
D=\left\{x \in \Omega: \sum_{i=1}^{k} \gamma_{i} e_{i}+\bar{s} \phi_{1}+(1-\bar{s}) \phi_{2}=0\right\} .
$$

Since $\left\|\phi_{1}, \phi_{2}\right\| \leq \delta \epsilon$ we derive from (2.28) that $\int_{\Omega}\left|\sum_{i=1}^{k} \gamma_{i} e_{i}\right|^{2} \leq \delta \epsilon$ for any $\epsilon \leq \epsilon_{0}$. Then $\sum_{i=1}^{k} \gamma_{i} e_{i}=0$ almost everywhere in $D$ with meas $D \geq \delta$. Since $\sum_{i=1}^{k} \gamma_{i} e_{i}$ is an eigenfunction of $-\Delta$ and $\gamma \neq 0$ we reach a contradiction. So (2.24) holds. Then we have that

$$
\begin{aligned}
I_{1}= & \int_{D_{1, \epsilon, s}}\left(\int_{0}^{1}\left[(1+\epsilon)\left|\sum_{i=1}^{k} \gamma_{i} e_{i}+s \phi_{1}+(1-s) \phi_{2}\right|^{\epsilon}-1\right] d s\right)^{2}\left|\phi_{1}-\phi_{2}\right|^{2} \\
\leq & \left(\int_{D_{1, \epsilon, s} \mid}\left|\int_{0}^{1}\left[(1+\epsilon)\left|\sum_{i=1}^{k} \gamma_{i} e_{i}+s \phi_{1}+(1-s) \phi_{2}\right|^{\epsilon}-1\right] d s\right|^{N}\right)^{\frac{2}{N}} \\
& \cdot\left(\int_{\left.D_{1, \epsilon, s}\left|\phi_{1}-\phi_{2}\right|^{\frac{2 N}{N-2}}\right)^{\frac{N-2}{N}}}\right. \\
\leq & C\left(\operatorname{meas}\left(D_{1, \epsilon, s}\right)\right)^{\frac{2}{N}}\left\|\phi_{1}-\phi_{2}\right\|=o(1)\left\|\phi_{1}-\phi_{2}\right\| .
\end{aligned}
$$

Estimate of $I_{2}$

Since $x \in D_{2, \epsilon, s}$ we have that

$$
\left|\sum_{i=1}^{k} \gamma_{i} e_{i}+s \phi_{1}+(1-s) \phi_{2}\right|^{\epsilon}-1 \rightarrow 0 .
$$

Hence

$$
\begin{aligned}
I_{2} & =\int_{D_{2, \epsilon, s}}\left(\int_{0}^{1}\left[(1+\epsilon)\left|\sum_{i=1}^{k} \gamma_{i} e_{i}+s \phi_{1}+(1-s) \phi_{2}\right|^{\epsilon}-1\right] d s\right)^{2}\left|\phi_{1}-\phi_{2}\right|^{2} \\
& \leq C\left(\left.\int_{D_{2, \epsilon, s}}\left|\int_{0}^{1}\left[(1+\epsilon)\left|\sum_{i=1}^{k} \gamma_{i} e_{i}+s \phi_{1}+(1-s) \phi_{2}\right|^{\epsilon}-1\right] d s\right|^{N}\right|^{\frac{2}{N}}\left\|\phi_{1}-\phi_{2}\right\|\right. \\
& =o(1)\left\|\phi_{1}-\phi_{2}\right\| .
\end{aligned}
$$

by the dominated convergence theorem. 


\section{Estimate of $I_{3}$}

Let us remark that if $x \in D_{3, \epsilon, s}$ then $\left|s \phi_{1}(x)+(1-s) \phi_{2}(x)\right| \geq\left\|\sum_{i=1}^{k} \gamma_{i} e_{i}\right\|_{\infty}$. Hence, using that $\left\|\phi_{1}, \phi_{2}\right\| \leq \delta \epsilon$ we get

$$
\text { meas }\left(D_{3, \epsilon, s}\right) \rightarrow 0 \quad \text { as } \epsilon \rightarrow 0 \forall s \in[0,1] .
$$

Moreover, for $x \in D_{3, \epsilon, s}$ we derive that

$$
|(1+\epsilon)| \sum_{i=1}^{k} \gamma_{i} e_{i}+s \phi_{1}+\left.(1-s) \phi_{2}\right|^{\epsilon}-1 \mid \leq C\left(1+\left|\phi_{1}\right|^{\frac{2}{N}}+\left|\phi_{2}\right|^{\frac{2}{N}}\right) .
$$

Let us estimate $I_{3}$,

$$
\begin{aligned}
I_{3} & =\int_{D_{3, \epsilon, s}}\left(\int_{0}^{1}\left[(1+\epsilon)\left|\sum_{i=1}^{k} \gamma_{i} e_{i}+s \phi_{1}+(1-s) \phi_{2}\right|^{\epsilon}-1\right] d s\right)^{2}\left|\phi_{1}-\phi_{2}\right|^{2} \\
& \leq C\left[\operatorname{meas}\left(D_{3, \epsilon, s}\right)^{\frac{2}{N}}+\left(\int_{D_{3, \epsilon, s}}\left|\phi_{1}\right|\right)^{\frac{2}{N}}+\left(\int_{D_{3, \epsilon, s}}\left|\phi_{2}\right|\right)^{\frac{2}{N}}\right]\left(\int_{D_{3, \epsilon, S}}\left|\phi_{1}-\phi_{2}\right|^{\frac{2 N}{N-2}}\right)^{\frac{N-2}{N}} \\
& =o(1)\left\|\phi_{1}-\phi_{2}\right\|
\end{aligned}
$$

Finally, from (2.29), (2.31), (2.34) we have that (2.18) becomes

$$
\left\|F\left(\phi_{1}\right)-F\left(\phi_{2}\right)\right\| \leq C(1+\epsilon) o(1)\left\|\phi_{1}-\phi_{2}\right\| \leq \frac{1}{2}\left\|\phi_{1}-\phi_{2}\right\|
$$

which proves that $F$ is a contraction from $B_{\epsilon}$ into itself with $\delta=2 C_{0}$. Hence the contraction mapping theorem provides the existence of a unique fixed point for the operator $F$ which gives the claim.

Remark 2.2. From the computation in Step 1 it follows that the constant $\delta$ is independent of $\gamma$ (it only depends on $\gamma_{0}$ ).

The proof of the previous Proposition does not hold if $\gamma=0$. Our aim is extend the definition of $\phi_{\epsilon}=\phi_{\epsilon}\left(a_{1}, \ldots, a_{k}, x\right)$ in order to cover this case.

Let us consider a sequence $\gamma_{n} \rightarrow 0, \gamma_{n} \neq 0$ and set $\phi_{\epsilon, n}=\phi_{\epsilon}\left(\gamma_{n}, x\right)$. By Remark 2.2 we deduce that there exists a subsequence $\gamma_{n}$ (denoted again by $\gamma_{n}$ ) and a function $\bar{\phi}_{\epsilon}$ such that

$$
\phi_{\epsilon, n} \rightarrow \bar{\phi}_{\epsilon} \text { as } n \rightarrow+\infty
$$

weakly in $H^{2}(\Omega) \cap H_{0}^{1}(\Omega)$ and strongly in $L^{2}(\Omega)$. If we show that $\bar{\phi}_{\epsilon} \equiv 0$ we can define

$$
\phi_{\epsilon}(0, x)=0 \text {. }
$$

It will be proved in next lemma. 
Lemma 2.3. We have that $\bar{\phi}_{\epsilon} \equiv 0$.

Proof. Let us write down the equation satisfied by $\phi_{\epsilon, n}$,

$$
\left\{\begin{array}{rlrl}
-\Delta \phi_{\epsilon, n}-\lambda \phi_{\epsilon, n}-\lambda\left[\left|\left(\sum_{i=1}^{k} \gamma_{i} e_{i}+\phi_{\epsilon, n}\right)\right|^{\epsilon}\left(\sum_{i=1}^{k} \gamma_{i} e_{i}+\phi_{\epsilon, n}\right)\right. & & \\
& \left.-\sum_{i=1}^{k} \gamma_{i} e_{i}+\phi_{\epsilon, n}\right]=\sum_{i=1}^{k} C_{i, n, \epsilon} e_{i} & & \text { in } \Omega \\
\sum_{i=1}^{k} \gamma_{i} e_{i}+\phi_{\epsilon, n}=0 & & \text { on } \partial \Omega .
\end{array}\right.
$$

Let us show that (up to a subsequence)

$$
C_{i, n, \epsilon} \rightarrow C_{i, \epsilon} \quad \text { as } n \rightarrow+\infty \quad \text { for any } i=1, . ., k .
$$

To prove (2.39) it is enough to multiply (2.38) by $e_{j}$ and integrate. We get

$$
C_{j, n, \epsilon} \int_{\Omega} e_{j}^{2} \leq \lambda \int_{\Omega}\left(\left|\sum_{i=1}^{k} \gamma_{i} e_{i}(x)+\phi_{\epsilon, n}\right|^{1+\epsilon}+\left|\sum_{i=1}^{k} \gamma_{i} e_{i}(x)+\phi_{\epsilon, n}\right|\right)
$$

and since $\gamma_{i, n}$ are bounded and $\left\|\phi_{\epsilon, n}\right\| \leq \delta \epsilon$ we get (2.39). Passing to the limit in (2.38) as $n \rightarrow+\infty$ we get that $\bar{\phi}_{\epsilon}$ satisfies

$$
\left\{\begin{array}{l}
-\Delta \bar{\phi}_{\epsilon}-\lambda\left|\bar{\phi}_{\epsilon}\right|^{\epsilon} \bar{\phi}_{\epsilon}=\sum_{i=1}^{k} C_{i, \epsilon} e_{i}(x) \text { in } \Omega \\
\bar{\phi}_{\epsilon}=0 \quad \text { on } \partial \Omega .
\end{array}\right.
$$

We argue by contradiction and suppose that $\bar{\phi}_{\epsilon} \not \equiv 0$. Define

$$
\psi_{\epsilon}=\frac{\bar{\phi}_{\epsilon}}{\left\|\bar{\phi}_{\epsilon}\right\|_{L^{q}(\Omega)}}
$$

for some $2<q<\frac{2 N}{N-2}$. Let us show that $\int_{\Omega}\left|\nabla \psi_{\epsilon}\right|^{2} \leq C$ where $C$ is a positive constant independent of $\epsilon$. Multiplying (2.41) by $\psi_{\epsilon}$ and using that $\int_{\Omega} \psi_{\epsilon} e_{i}=0$ for any $i=1, . ., k$ we have

$$
\int_{\Omega}\left|\nabla \psi_{\epsilon}\right|^{2}=\lambda \int_{\Omega}\left|\bar{\phi}_{\epsilon}\right|^{\epsilon} \psi_{\epsilon}^{2} \leq\left(\int_{\Omega}\left|\bar{\phi}_{\epsilon}\right|^{\frac{q \epsilon}{q-2}}\right)^{\frac{q-2}{q}}\left(\int_{\Omega}\left|\bar{\psi}_{\epsilon}\right|^{q}\right)^{\frac{2}{q}} \leq C .
$$

Hence we have that $\psi_{\epsilon} \rightarrow \psi_{0}$ weakly in $H_{0}^{1}(\Omega)$ and $\left\|\psi_{0}\right\|_{L^{q}(\Omega)}=1$. Finally, multiplying (2.41) by $e$, where $e$ is an eigenfunction of $-\Delta$ associated to $\mu \neq \lambda$ we get

$$
\mu \int_{\Omega} \psi_{\epsilon} e=\lambda \int_{\Omega}\left|\bar{\phi}_{\epsilon}\right|^{\epsilon} \psi_{\epsilon} e=\lambda \int_{\Omega}\left(1+\epsilon \log \left|\bar{\phi}_{\epsilon}\right| \int_{0}^{1}\left|\bar{\phi}_{\epsilon}\right|^{t \epsilon} d t\right) \psi_{\epsilon} e
$$


which implies

$$
\begin{aligned}
(\mu-\lambda) \int_{\Omega} \psi_{\epsilon} e & =\epsilon \lambda \int_{\Omega} \log \left|\bar{\phi}_{\epsilon}\right| \int_{0}^{1}\left|\bar{\phi}_{\epsilon}\right|^{t \epsilon} d t \psi_{\epsilon} e \\
& =\frac{\epsilon \lambda}{\left\|\bar{\phi}_{\epsilon}\right\|_{L^{q}(\Omega)}} \int_{\Omega} \log \left|\bar{\phi}_{\epsilon}\right| \int_{0}^{1}\left|\bar{\phi}_{\epsilon}\right|^{t \epsilon} d t \bar{\phi}_{\epsilon} e \\
& \left(\text { using that }\left.\left.|\log | \bar{\phi}_{\epsilon}\left|\int_{0}^{1}\right| \bar{\phi}_{\epsilon}\right|^{1+t \epsilon} d t|\leq C| \bar{\phi}_{\epsilon}\right|^{\frac{1}{2}}\right) \\
& \leq C\|e\|_{L^{\infty}(\Omega)} \frac{\epsilon \lambda}{\left\|\bar{\phi}_{\epsilon}\right\|_{L^{q}(\Omega)}} \int_{\Omega}\left|\bar{\phi}_{\epsilon}\right|^{\frac{1}{2}} \leq C \epsilon\left\|\bar{\phi}_{\epsilon}\right\|_{L^{q}(\Omega)} \leq C \epsilon .
\end{aligned}
$$

Passing to the limit in (2.45) we get $\int_{\Omega} \psi_{0} e=0$. Since we also have that $\int_{\Omega} \psi_{0} e_{i}=0$ for any $i=1, . ., k$ we derive that $\psi \equiv 0$ and this is not possible since $\left\|\psi_{0}\right\|_{L^{q}(\Omega)}=1$. So we reach a contradiction and then $\bar{\phi}_{\epsilon} \equiv 0$.

Corollary 2.4. For any $\gamma$ such that $|\gamma| \leq\left|\gamma_{0}\right|$ there exists a unique function $\phi_{\epsilon}=$ $\phi_{\epsilon}(\gamma, x) \in B_{\epsilon}$ which satisfies

$$
\left\{\begin{array}{cc}
-\Delta\left[\lambda_{k}^{\frac{1}{\epsilon}}\left(\sum_{i=1}^{k} \gamma_{i} e_{i}+\phi_{\epsilon}\right)\right]-\lambda\left|\left(\sum_{i=1}^{k} \gamma_{i} e_{i}+\phi_{\epsilon}\right)\right|^{\epsilon}\left[\lambda_{k}^{\frac{1}{\epsilon}}\left(\sum_{i=1}^{k} \gamma_{i} e_{i}+\phi_{\epsilon}\right)\right] & \text { in } \Omega \\
=\sum_{i=1}^{k} C_{i, \epsilon} e_{i} & \text { on } \partial \Omega \\
\lambda_{k}^{\frac{1}{\epsilon}}\left(\sum_{i=1}^{k} \gamma_{i} e_{i}+\phi_{\epsilon}\right)=0 &
\end{array}\right.
$$

for some constants $C_{i, \epsilon} \in \mathbb{R}$.

Proof. Writing down the equation satisfied by $\phi_{\epsilon}$ in (2.10) we get (2.48). The uniqueness of the function $\phi_{\epsilon}$ follows by the contraction mapping theorem (see the proof of Proposition 2.1).

We end this section proving some properties of the function $\phi_{\epsilon}(\gamma, x)$.

Lemma 2.5. The function $\phi_{\epsilon}(\gamma, x)$ satisfies

$$
\phi_{\epsilon}(-\gamma, x)=-\phi_{\epsilon}(\gamma, x)
$$

Proof. The claim is a consequence of the uniqueness of the function $\phi_{\epsilon}$. Indeed we have that $-\phi_{\epsilon}(-\gamma, x)$ satisfies the problem (2.48) and using again the uniqueness of the solution we get the claim. 
Lemma 2.6. The function $\phi_{\epsilon}(\gamma, x)$ is $C^{1}$ in $\gamma$ for $\gamma \neq 0$.

Proof. The claim follows by the implicit function theorem applied to $T_{\epsilon}(\gamma, u)$ : $\left(0, \gamma_{0}\right) \times W_{k}^{\perp} \cap H^{2}(\Omega) \cap H_{0}^{1}(\Omega) \rightarrow W_{k}^{\perp}$,

$$
T_{\epsilon}(\gamma, u)=\Pi^{\perp}\left[\Delta\left(\sum_{i=1}^{k} \gamma_{i} e_{i}+u\right)+\left|\sum_{i=1}^{k} \gamma_{i} e_{i}+u\right|^{\epsilon}\left(\sum_{i=1}^{k} \gamma_{i} e_{i}+u\right)\right]
$$

for $\epsilon \in\left(0, \epsilon_{0}\right)$. We have that $T_{\epsilon}\left(\gamma, \phi_{\epsilon}\right)=0$. If we show that $T_{\epsilon}^{\prime}\left(\gamma, \phi_{\epsilon}\right)$ is invertible, by the implicit function theorem we get the claim. We will show that

$$
\left\|T_{\epsilon}^{\prime}\left(\gamma, \phi_{\epsilon}\right)\right\| \geq C>0 \quad \text { for any } \epsilon \in\left(0, \epsilon_{0}\right) .
$$

By contradiction let us suppose that there exists a sequence $\epsilon_{n} \rightarrow 0, v_{n} \in W_{k}^{\perp} \cap$ $H^{2}(\Omega) \cap H_{0}^{1}(\Omega)$ such that

$$
\left\|v_{n}\right\|=1
$$

and

$$
\left\|T_{\epsilon_{n}}^{\prime}\left(\gamma, \phi_{\epsilon_{n}}\right) v_{n}\right\|_{L^{2}(\Omega)} \rightarrow 0 \quad \text { as } n \rightarrow+\infty .
$$

By (2.52) it follows that there exists $v_{0} \in W_{k}^{\perp} \cap H^{2}(\Omega) \cap H_{0}^{1}(\Omega)$ such that

$$
v_{n} \rightarrow v_{0}
$$

weakly in $H^{2}(\Omega)$ and strongly in $L^{2}(\Omega)$. Arguing as in Step 2 of Proposition 2.1 we can pass to the limit in (2.53) and then

$$
\left\|\Pi^{\perp}\left(\Delta v_{0}+\lambda v_{0}\right)\right\|_{L^{2}(\Omega)}=0 .
$$

This implies that $v_{0}=0$. On the other hand, by (2.53) we get

$$
\int_{\Omega}\left|\Pi^{\perp}\left(\Delta v_{n}+\lambda\left(1+\epsilon_{n}\right)\left|\sum_{i=1}^{k} \gamma_{i} e_{i}+\phi_{\epsilon_{n}}\right| v_{n}\right)\right|^{2}=o(1)
$$

and since $\Delta v_{n} \in W_{k}^{\perp}$ and $v_{n} \rightarrow 0$ in $L^{2}(\Omega)$ we have

$$
\int_{\Omega}\left|\Delta v_{n}\right|^{2}+o(1)=o(1)
$$

a contradiction with (2.52). So $T_{\epsilon}^{\prime}\left(\gamma, \phi_{\epsilon}\right)$ is an invertible operator and the claim follows. 


\section{The reduced problem}

In this section we prove Theorem 1.3. Let $J_{\epsilon}(u): H_{0}^{1}(\Omega) \rightarrow R$ defined by

$$
J_{\epsilon}(u)=\frac{1}{2} \int_{\Omega}|\nabla u|^{2}-\frac{1}{2+\epsilon} \int_{\Omega}|u|^{2+\epsilon} .
$$

It is well known that critical points of (3.1) provides solutions to (1.2). For $|\gamma| \leq$ $\gamma_{0}$ let us consider the function $\phi_{\epsilon}=\phi_{\epsilon}(\gamma, x)$ defined in Corollary 2.4. Using the same notation of the previous section let us introduce the reduced functional $I_{\epsilon}(\Gamma):\left\{\Gamma \in \mathbb{R}^{k}:|\Gamma| \leq \gamma_{0}\right\} \rightarrow \mathbb{R}$ defined by

$$
I_{\epsilon}(\Gamma)=J_{\epsilon}\left[\lambda \lambda^{\frac{1}{\epsilon}}\left(\sum_{i=1}^{k} \Gamma_{i} e_{i}+\phi_{\epsilon}(\Gamma, x)\right)\right] .
$$

We have the following:

Lemma 3.1. A function $u_{\epsilon}=\lambda^{\frac{1}{\epsilon}}\left(\sum_{i=1}^{k} \gamma_{i} e_{i}+\phi_{\epsilon}(\gamma, x)\right)$ is a solution to (1.2) if and only if $\gamma=\left(\gamma_{1}, . ., \gamma_{k}\right) \in \mathbb{R}^{k}$ is a critical point to $I_{\epsilon}$.

Proof. If $u_{\epsilon}$ is a solution to (1.2) we have that $J_{\epsilon}^{\prime}\left(u_{\epsilon}\right)=0$ and then

$$
\frac{\partial I_{\epsilon}(\gamma)}{\partial a_{m}}=J_{\epsilon}^{\prime}\left(u_{\epsilon}\right) \lambda^{\frac{1}{\epsilon}}\left(e_{m}+\frac{\partial \phi_{\epsilon}(\gamma, x)}{\partial a_{m}}\right)=0 .
$$

Conversely, let us suppose that $\gamma$ is a critical point to $I_{\epsilon}$. Differentiating again (3.19) we derive

$$
0=\frac{\partial I_{\epsilon}(\gamma)}{\partial a_{m}}=J_{\epsilon}^{\prime}\left(\sum_{i=1}^{k} \gamma_{i} e_{i}+\phi_{\epsilon}(\gamma, x)\right)\left(e_{m}+\frac{\partial \phi_{\epsilon}(\gamma, x)}{\partial a_{m}}\right) .
$$

From (2.48) we get that

$$
J_{\epsilon}^{\prime}\left(\sum_{i=1}^{k} \gamma_{i} e_{i}+\phi_{\epsilon}(\gamma, x)\right) \in W_{k}
$$

and then

$$
J_{\epsilon}^{\prime}\left(\sum_{i=1}^{k} \gamma_{i} e_{i}+\phi_{\epsilon}(\gamma, x)\right)\left(\frac{\partial \phi_{\epsilon}(\gamma, x)}{\partial a_{m}}\right)=0 .
$$

Then (3.4) becomes

$$
0=J_{\epsilon}^{\prime}\left(\sum_{i=1}^{k} \gamma_{i} e_{i}+\phi_{\epsilon}(\gamma, x)\right)\left(e_{m}\right) .
$$


From (3.7) we get that $J_{\epsilon}^{\prime}\left(\sum_{i=1}^{k} \gamma_{i} e_{i}+\phi_{\epsilon}(\gamma, x)\right) \in W_{k}^{\perp}$ and then we have

$$
J_{\epsilon}^{\prime}\left(\sum_{i=1}^{k} \gamma_{i} e_{i}+\phi_{\epsilon}(\gamma, x)\right)=0
$$

which gives the claim.

Next result concerns the expansion of the reduced functional.

Lemma 3.2. We have that

$$
I_{\epsilon}(\Gamma)=\frac{\lambda^{1+\frac{2}{\epsilon}}}{4} \epsilon\left[\int_{\Omega}\left|\left(\sum_{i=1}^{k} \Gamma_{i} e_{i}\right)\right|^{2}\left(2 \log \left|\sum_{i=1}^{k} \Gamma_{i} e_{i}\right|-1\right)+O(\epsilon)\right]
$$

uniformly with respect to $\Gamma$ as $\epsilon \rightarrow 0$.

Proof. We have

$$
\begin{aligned}
\frac{I_{\epsilon}(\Gamma)}{\lambda^{\frac{2}{\epsilon}}} & =\frac{1}{2} \int_{\Omega}\left|\nabla\left(\sum_{i=1}^{k} \Gamma_{i} e_{i}+\phi_{\epsilon}\right)\right|^{2}-\frac{\lambda}{2+\epsilon} \int_{\Omega}\left|\sum_{i=1}^{k} \Gamma_{i} e_{i}+\phi_{\epsilon}\right|^{2+\epsilon} \\
& =\frac{1}{2} \int_{\Omega}\left[\lambda\left(\sum_{i=1}^{k} \Gamma_{i} e_{i}\right)^{2}+2 \nabla\left(\sum_{i=1}^{k} \Gamma_{i} e_{i}\right) \cdot \nabla \phi_{\epsilon}+\left|\nabla \phi_{\epsilon}\right|^{2}\right] \\
& -\lambda\left(\frac{1}{2}-\frac{\epsilon}{4}+O\left(\epsilon^{2}\right)\right) \int_{\Omega}\left[\left(\sum_{i=1}^{k} \Gamma_{i} e_{i}\right)^{2}+2\left(\sum_{i=1}^{k} \Gamma_{i} e_{i}\right) \phi_{\epsilon}+\phi_{\epsilon}^{2}\right] \\
& \cdot\left[1+\epsilon \log \left|\sum_{i=1}^{k} \Gamma_{i} e_{i}+\phi_{\epsilon}\right| \int_{0}^{1}\left|\sum_{i=1}^{k} \Gamma_{i} e_{i}+\phi_{\epsilon}\right|^{t \epsilon} d t\right] .
\end{aligned}
$$

Recalling that $\phi_{\epsilon} \in W_{k}^{\perp}$ we have that $\int_{\Omega} \nabla\left(\sum_{i=1}^{k} \Gamma_{i} e_{i}\right) \cdot \nabla \phi_{\epsilon}=0$. Moreover since $\left\|\phi_{\epsilon}\right\| \leq \delta \epsilon$ with $\delta$ independent of $\Gamma$ we get that

$$
\begin{aligned}
& \frac{I_{\epsilon}(\Gamma)}{\lambda^{\frac{2}{\epsilon}}} \\
& =\lambda \int_{\Omega}\left[\frac{\epsilon}{2}\left(\sum_{i=1}^{k} \Gamma_{i} e_{i}\right)^{2} \log \left|\sum_{i=1}^{k} \Gamma_{i} e_{i}\right| \int_{0}^{1}\left|\sum_{i=1}^{k} \Gamma_{i} e_{i}+\phi_{\epsilon}\right|^{t \epsilon} d t-\frac{\epsilon}{4}\left(\sum_{i=1}^{k} \Gamma_{i} e_{i}\right)^{2}\right] \\
& \quad+O\left(\epsilon^{2}\right) .
\end{aligned}
$$


Since the function $x^{2}|x|^{t \epsilon} \rightarrow x^{2}$ uniformly on the compact set as $\epsilon \rightarrow 0$ for any $t \in[0,1]$, arguing as in Step 2 of Proposition 2.1, we get

$$
\left(\sum_{i=1}^{k} \Gamma_{i} e_{i}\right)^{2} \int_{0}^{1}\left|\sum_{i=1}^{k} \Gamma_{i} e_{i}+\phi_{\epsilon}\right|^{t \epsilon} d t \rightarrow\left(\sum_{i=1}^{k} \Gamma_{i} e_{i}\right)^{2}
$$

and the claim follows.

The main result of this section follows by a classical result of critical point theory due to Clark ( [2]). Here we mention a slightly different statement (see [7]).

Theorem 3.3. Let $E$ a Banach space, $I \in C^{1}(E, \mathbb{R})$ with $I$ bounded from below and satisfying the Palais-Smale condition. Suppose $I(0)=0$, there is a set $K \subset E$ such that $K$ is homeomorphic to $S^{j-1}$ by an odd map and $\sup _{K} I<0$. Then $I$ possesses at least $j$ distinct pairs of critical points.

Remark 3.4. From the proof of previous theorem it is possible to see that the critical points $x$ of $I$ satisfy $I(x)<0$. Hence it is enough to require the differentiability of the functional just for $x \neq 0$ and we only need the validity of the Palais-Smale condition for negative values of $I$.

We will apply Theorem 3.3 to find at least $j$ pairs of critical points to the functional $I_{\epsilon}$ for $\epsilon$ small enough.

Proposition 3.5. For $\gamma_{0}$ sufficiently large and for $\epsilon$ small enough the functional $I_{\epsilon}$ admits at least $j$ distinct pairs of critical points.

Proof. We will apply Theorem 3.3 with $I=\frac{4}{\epsilon \lambda^{\frac{2}{\epsilon}}} I_{\epsilon}$ and $E=\left\{\gamma \in \mathbb{R}^{k}:|\Gamma| \leq \gamma_{0}\right\}$. Note that by Lemma 2.5 we have that $I_{\epsilon}$ is an even functional and by Lemma 2.6 it is differentiable in $E \backslash\{0\}$. Let us show that $I$ is bounded from below. By Lemma 3.2 it is enough to show that the functional

$$
I_{0}(\Gamma)=\int_{\Omega}\left|\left(\sum_{i=1}^{k} \Gamma_{i} e_{i}\right)\right|^{2}\left(2 \log \left|\sum_{i=1}^{k} \Gamma_{i} e_{i}\right|-1\right)
$$

is bounded from below. Since the function $g(t)=t^{2}(2 \log |t|-1)$ satisfies $g(t) \geq$ -1 we derive that

$$
\left|I_{0}(\Gamma)\right| \geq- \text { meas } \Omega
$$

which gives the claim.

Let us prove that $I$ satisfies the Palais-Smale condition. Since $I$ is defined for $|\Gamma| \leq\left|\gamma_{0}\right|$ we have to check any Palais-Smale sequence is far away from the sphere $|\Gamma|=\gamma_{0}$. By Remark 3.4 this leads to prove that

$$
I(\Gamma)>0 \quad \text { if }|\Gamma|=\gamma_{0} .
$$


We will show that (3.16) is verified if $\left|\gamma_{0}\right|$ is large enough. By Lemma 3.2 it is enough to prove

$$
I_{0}(\Gamma)=\int_{\Omega}\left|\left(\sum_{i=1}^{k} \Gamma_{i} e_{i}\right)\right|^{2}\left(2 \log \left|\sum_{i=1}^{k} \Gamma_{i} e_{i}\right|-1\right)>0 \quad \text { if }|\Gamma|=\left|\gamma_{0}\right| .
$$

Let $\Gamma$ satisfying $|\Gamma|=\left|\gamma_{0}\right|$ and suppose that $\Gamma_{1}=\max _{i=1, \ldots, k} \Gamma_{i}$. We have that $\Gamma_{1} \geq$ $\frac{\left|\gamma_{0}\right|}{\sqrt{N}}$ and

$$
\begin{aligned}
I_{0}(\Gamma) & =2 \int_{\Omega}\left(\sum_{i=1}^{k} \Gamma_{i} e_{i}\right)^{2} \log \left|\sum_{i=1}^{k} \Gamma_{i} e_{i}\right|-\gamma_{0}^{2} \\
& =2 \log \left|\Gamma_{1}\right| \gamma_{0}^{2}+2 \int_{\Omega}\left(\sum_{i=1}^{k} \Gamma_{i} e_{i}\right)^{2} \log \left|e_{1}+\sum_{i=2}^{k} \frac{\Gamma_{i}}{\Gamma_{1}} e_{i}\right|-\gamma_{0}^{2} \\
& =|\gamma|^{2}\left[2 \log \left|\Gamma_{1}\right|+2 \int_{\Omega}\left(\sum_{i=1}^{k} \frac{\gamma_{i}}{\gamma_{0}} e_{i}\right)^{2} \log \left|e_{1}+\sum_{i=2}^{k} \frac{\gamma_{i}}{\Gamma_{1}} e_{i}\right|-1\right]
\end{aligned}
$$

and since $t^{2} \log t \geq-\frac{1}{2} e^{-\frac{1}{4}}$ (3.18) becomes

$$
I_{0} \geq \gamma_{0}^{2}\left[2 \log \left|\Gamma_{1}\right|-e^{-\frac{1}{4}} \text { meas } \Omega-1\right] \geq \gamma_{0}^{2}\left[2 \log \frac{\left|\gamma_{0}\right|}{\sqrt{N}}-e^{-\frac{1}{4}} \text { meas } \Omega-1\right] .
$$

Choosing

$$
\left|\gamma_{0}\right|>\exp \left\{\sqrt{N} \frac{e^{-\frac{1}{4}} \text { meas } \Omega+1}{2}\right\}
$$

we have that $I_{0}(\Gamma)>0$. So the Palais-Smale condition is satisfied.

We end our proof by showing that there exists a sphere $K \subset E$ such that $\sup _{K} I<0$. To do this let us observe that the function $g(t)=t^{2}(2 \log |t|-1)$ satisfies $g(t)<0$ if $|t|<e^{\frac{1}{2}}$.

Hence, choosing $|\Gamma|=\frac{e^{\frac{1}{2}}}{2 \sum_{i=1}^{k}\left\|e_{i}\right\|_{\infty}}$ we deduce that $\left|\sum_{i=1}^{k} e_{i}\right|<\frac{1}{2} e^{\frac{1}{2}}$ and then

$$
\int_{\Omega}\left(\sum_{i=1}^{k} \Gamma_{i} e_{i}\right)^{2}\left(2 \log \left|\sum_{i=1}^{k} \Gamma_{i} e_{i}\right|-1\right)<0,
$$

which gives the claim.

Proof of Theorem 1.3. It follows by Lemma 3.1 and Proposition 3.5. 


\section{The asymptotic behavior of the solution}

In this section we study problem (1.2) and, under some suitable conditions, we try to show that the set of its solution is the same prescribed in the previous section.

Proof of Theorem 1.4. Set $v_{n}=\frac{u_{n}}{\left\|u_{n}\right\|_{2}}$. Then $v_{n}$ solves

$$
\begin{cases}-\Delta v_{n}=\left\|u_{n}\right\|_{2}^{\epsilon_{n}}\left|v_{n}\right|^{\epsilon} v_{n} & \text { in } \Omega \\ \left\|v_{n}\right\|_{2}=1 & \text { on } \partial \Omega . \\ v_{n}=0 & \end{cases}
$$

By assumption we have that, up some subsequence again denoted by $\epsilon_{n} \rightarrow 0$,

$$
\left\|u_{n}\right\|_{2}^{\epsilon_{n}} \rightarrow \lambda \text {. }
$$

Moreover, by mean value theorem we get

$$
\left|v_{n}\right|^{\epsilon_{n}} v_{n}=v_{n}+\epsilon_{n} v_{n} \log \left|v_{n}\right| \int_{0}^{1}\left|v_{n}\right|^{\epsilon_{n}} d t
$$

Since $\left\|v_{n}\right\|_{2}=1$ we get that $v_{n} \log \left|v_{n}\right| \int_{0}^{1}\left|v_{n}\right|^{t \epsilon_{n}} d t$ is bounded in $L^{1}(\Omega)$. Hence using the standard regularity theory we get the existence of $v_{0} \in C^{2}(\Omega)$ such that

$$
\left|v_{n}\right|^{\epsilon_{n}} v_{n} \rightarrow v_{0} \quad \text { in } L^{1}(\Omega)
$$

and

$$
\begin{cases}-\Delta v_{0}=\lambda v_{0} & \text { in } \Omega \\ \left\|v_{0}\right\|_{2}=1 & \\ v_{0}=0 & \text { on } \partial \Omega .\end{cases}
$$

Since $\left\|v_{0}\right\|_{2}=1$ we derive from (4.5) that $\lambda \in \sigma(-\Delta)$ and then (1.7) follows by (4.2). Moreover, again by (4.5) we have that

$$
v_{0}=\sum_{i=1}^{m(\lambda)} \gamma_{i} e_{i}^{(\lambda)}
$$

which gives (1.8)

Let us show that $\gamma_{i}$ is a critical point of the function (1.4). Setting $z_{n}=\frac{u_{n}}{\lambda^{\frac{1}{\epsilon_{n}}}}$ we have that $z_{n}$ satisfy

$$
\left\{\begin{array}{ll}
-\Delta z_{\epsilon}=\lambda\left|z_{\epsilon}\right|^{\epsilon} z_{\epsilon} & \text { in } \Omega \\
z_{\epsilon}=0 & \text { on } \partial \Omega
\end{array} .\right.
$$

Multiplying (4.7) by $e_{j} \in W_{k}$ we get

$$
\lambda \int_{\Omega} z_{n} e_{j}=\lambda \int_{\Omega}\left|z_{n}\right|^{r \epsilon_{n}} z_{n} e_{j}=\lambda \int_{\Omega}\left(1+\epsilon_{n} \log \left|z_{n}\right| \int_{0}^{1}\left|z_{n}\right|^{t \epsilon_{n}} d t\right) z_{n} e_{j}
$$


which implies

$$
0=\int_{\Omega} \log \left|z_{n}\right| \int_{0}^{1}\left|z_{n}\right|^{t \epsilon_{n}} d t z_{n} e_{j} .
$$

From (4.9) we derive

$$
0=\int_{\Omega}\left(\log \frac{\left|u_{n}\right|}{\left\|u_{n}\right\|_{2}}+\log \frac{\left\|u_{n}\right\|_{2}}{\lambda^{\epsilon_{n}}}\right) \int_{0}^{1} \frac{\left|u_{n}\right|^{t \epsilon_{n}}}{\left\|u_{n}\right\|_{2}^{t \epsilon_{n}}} \frac{\left\|u_{n}\right\|_{2}^{t \epsilon_{n}}}{\lambda^{t}} d t \frac{u_{n}}{\left\|u_{n}\right\|_{2}} e_{j} .
$$

Since $\frac{u_{n}}{\left\|u_{n}\right\|_{2}} \rightarrow \sum_{i=1}^{k} \gamma_{i} e_{i}$ and meas $\left\{x \in \Omega: e_{i}(x)=0\right\}=0$ for any $i=1, \ldots, k$, we get that $\frac{\left|u_{n}\right|^{t \epsilon_{n}}}{\left\|u_{n}\right\|_{2}^{t \epsilon_{n}}} \rightarrow 1$ as $\epsilon_{n} \rightarrow 0$ for any $t \in[0,1]$ and almost everywhere in $\Omega$. Passing to the limit in (4.10) we have

$$
0=\int_{\Omega} \log \left|\sum_{i=1}^{k} \gamma_{i} e_{i}\right| \sum_{i=1}^{k} \gamma_{i} e_{i} e_{j} .
$$

Observing that $\int_{\Omega} \log \left|\sum_{i=1}^{k} \gamma_{i} e_{i}\right| \sum_{i=1}^{k} \gamma_{i} e_{i} e_{j}=\left.\frac{\partial J(\Gamma)}{\partial \gamma_{j}}\right|_{\Gamma=\gamma}$ we get that $\left(\gamma_{1}, \ldots, \gamma_{m}(\lambda)\right)$ is a critical point of the functional (1.4).

In order to finish our proof we have to prove (1.8) (note that our argument only gives that

$$
u_{n}=(\lambda+o(1))^{\frac{1}{\epsilon_{n}}}\left(\sum_{i=1}^{m(\lambda)} \gamma_{i} e_{i}^{(\lambda)}+\phi_{\epsilon}\right)
$$

that is a weaker statement that (1.8).

In order to show (1.8) we need the following estimate,

$$
\left\|u_{n}\right\|_{2}^{\epsilon_{n}}-\lambda=o\left(\epsilon_{n}\right) .
$$

To prove (4.13) let us multiply (4.1) by $e_{j} \in W_{k}$ and integrate. Here $j$ is chosen so that $a_{j} \neq 0$. We have

$$
\begin{aligned}
\lambda \int_{\Omega} v_{n} e_{j} & =\left(\left\|u_{n}\right\|_{2}^{\epsilon_{n}}-\lambda\right) \int_{\Omega}\left|v_{n}\right|^{\epsilon_{n}} v_{n} e_{j}+\lambda \int_{\Omega}\left|v_{n}\right| \epsilon_{n} v_{n} e_{j} \\
& =\left(\left\|u_{n}\right\|_{2}^{\epsilon_{n}}-\lambda\right) \int_{\Omega}\left|v_{\epsilon}\right| \epsilon_{n} v_{\epsilon} e_{j}+\lambda \int_{\Omega}\left(1+\epsilon_{n} \log \left|v_{n}\right|\right) v_{n} e_{j}+O\left(\epsilon_{n}^{2}\right),
\end{aligned}
$$

which implies

$$
0=\frac{\left\|u_{n}\right\|_{2}^{\epsilon_{n}}-\lambda}{\epsilon_{n}} \int_{\Omega}\left|v_{n}\right| v_{n} e_{j}+\lambda \int_{\Omega} \log \left|v_{n}\right| v_{n} e_{j}+O\left(\epsilon_{n}\right) .
$$

Passing to the limit in (4.15) and using (4.11) we get

$$
a_{j} \lim _{n \rightarrow+\infty} \frac{\left\|u_{n}\right\|_{2}^{\epsilon_{n}}-\lambda}{\epsilon_{n}}=0
$$

and since $a_{j} \neq 0$ we derive (4.13).

So from (4.13) we get that (4.12) becomes (1.8). 


\section{The uniqueness result}

In this section we prove Theorem 1.5 and its corollaries.

Proof of Theorem 1.5. From Theorem 1.3 we have the existence of at least $\sum_{i=1}^{\bar{\lambda}} m\left(\lambda_{i}\right)$ pairs of nontrivial solutions to (1.2). By contradiction let us suppose that there exists another solution $v_{\epsilon}$ satisfying (1.9). From Theorem 1.4 we have that, for some sequence $\epsilon_{n} \rightarrow 0$, there exists $\lambda \in \sigma(-\Delta)$ such that

$$
\left(\int_{\Omega}\left|v_{\epsilon_{n}}\right|^{2}\right)^{\epsilon_{n}} \rightarrow \lambda \in \sigma(-\Delta), \quad \text { as } n \rightarrow+\infty .
$$

Corresponding to this eigenvalue $\lambda$ we have that (again by Theorem 1.3) there exist at least $k=m(\lambda)$ pairs of nontrivial solutions $\pm u_{\epsilon, 1}, . ., \pm u_{\epsilon, k}$ verifying (1.3). In this way we have constructed a solution $v_{\epsilon_{n}}=v_{n}$ different from $\pm u_{\epsilon, 1}, . ., \pm u_{\epsilon, k}$ such that $v_{n}=\lambda^{\frac{1}{\epsilon_{n}}}\left(\sum_{i=1}^{k} \gamma_{i}^{(j)} e_{i}+\phi_{n}\right)$ with $\phi_{n} \rightarrow 0$ in $C^{2}(\Omega)$ for some $j=1, \ldots, k$. It is not restrictive to assume that $j=1$. Set $u_{\epsilon_{n}, 1}=u_{n}$. Since $v_{n} \not \equiv u_{n}$ we can define

$$
w_{n}=\frac{v_{n}-u_{n}}{\left\|v_{n}-u_{n}\right\|_{L^{2}(\Omega)}}
$$

which satisfies

$$
\begin{cases}-\Delta w_{n}=\left(1+\epsilon_{n}\right) \int_{0}^{1}\left|t v_{n}+(1-t) u_{n}\right|^{\epsilon_{n}} d t w_{n} & \text { in } \Omega \\ w_{n}=0 & \text { on } \partial \Omega \\ \left\|w_{n}\right\|_{L^{2}(\Omega)}=1 & \end{cases}
$$

By (1.8) and since meas $\left\{x \in \Omega: e_{i}(x)=0\right\}=0$ for any $i=1, . ., k$ we get that $\left|v_{n}\right|^{\epsilon_{n}} \rightarrow \lambda,\left|u_{n}\right|^{\epsilon_{n}} \rightarrow \lambda$ almost everywhere in $\Omega$. Then,

$$
\int_{0}^{1}\left|t v_{n}+(1-t) u_{n}\right|^{\epsilon_{n}} d t \rightarrow \lambda \quad \text { almost everywhere in } \Omega .
$$

From the standard regularity theory we get that there exists $w \in H_{0}^{1}(\Omega)$ such that $w_{n} \rightarrow w$ weakly in $H_{0}^{1}(\Omega)$ and $w$ satisfies

$$
\begin{cases}-\Delta w=\lambda w & \text { in } \Omega \\ w=0 & \text { on } \partial \Omega\end{cases}
$$

Hence $w=\sum_{i=1}^{k} \beta_{i} e_{i}$. Next step is to prove that $\beta_{i}=0$ for any $i=1, . ., k$. To show 
it let us multiply (5.3) by $e_{j}, j=1, . ., k$ and integrate. We have

$$
\begin{aligned}
\lambda \int_{\Omega} w_{n} e_{j}= & \lambda\left(1+\epsilon_{n}\right) \int_{\Omega} \int_{0}^{1}\left|t \frac{v_{n}}{\lambda^{\frac{1}{\epsilon_{n}}}}+(1-t) \frac{u_{n}}{\lambda^{\frac{1}{\epsilon_{n}}}}\right|^{\epsilon_{n}} d t w_{n} e_{j} \\
= & \lambda\left(1+\epsilon_{n}\right) \int_{\Omega}\left(1+\epsilon_{n} \int_{0}^{1} \int_{0}^{1}\left|t \frac{v_{n}}{\lambda^{\frac{1}{\epsilon_{n}}}}+(1-t) \frac{u_{n}}{\lambda^{\frac{1}{\epsilon_{n}}}}\right|^{\epsilon_{n} s} d s\right. \\
& \left.\cdot \log \left|t \frac{v_{n}}{\lambda^{\frac{1}{\epsilon_{n}}}}+(1-t) \frac{u_{n}}{\lambda^{\frac{1}{\epsilon_{n}}}}\right| d t\right) w_{n} e_{j}
\end{aligned}
$$

which implies

$$
\begin{aligned}
& 0=\int_{\Omega}(1+\int_{0}^{1} \int_{0}^{1}\left|t \frac{v_{n}}{\lambda^{\frac{1}{\epsilon_{n}}}}+(1-t) \frac{u_{n}}{\lambda^{\frac{1}{\epsilon_{n}}}}\right|^{\epsilon_{n} s} d s \\
&\left.\cdot \log \left|t \frac{v_{n}}{\lambda^{\frac{1}{\epsilon_{n}}}}+(1-t) \frac{u_{n}}{\lambda^{\frac{1}{\epsilon_{n}}}}\right| d t\right) w_{n} e_{j}+O(\epsilon)
\end{aligned}
$$

and passing to the limit we get

$$
\sum_{i=1}^{k} \beta_{i} \int_{\Omega}\left(1+\log \left|\sum_{l=1}^{k} \gamma_{l}^{(1)} e_{l}\right|\right) e_{i} e_{j}=0 .
$$

A straight-forward computation shows that

$$
\frac{\partial^{2} J\left(\gamma^{(1)}\right)}{\partial \gamma_{i} \partial \gamma_{j}}=2 \int_{\Omega}\left(1+\log \left|\sum_{i=1}^{k} \gamma_{i}^{(1)} e_{i}\right|\right) e_{i} e_{j}
$$

By the non-degeneracy of the critical point $\gamma^{(1)}$ we get that the matrix

$$
\int_{\Omega}\left(1+\log \left|\sum_{i=1}^{k} \bar{\gamma}_{i}^{(1)} e_{i}\right|\right) e_{i} e_{j}
$$

is invertible and then the linear system (5.8) admits only the trivial solution $\beta_{i}=0$ for any $i=1, \ldots, k$. Hence $w \equiv 0$. Last step of our proof is to show that $w \equiv 0$ leads to a contradiction.. Multiplying (5.3) by $w_{n}$ we get

$$
\int_{\Omega}\left|\nabla w_{n}\right|^{2} \leq C \int_{\Omega}\left|w_{n}\right|^{2} .
$$

Then $w_{n} \rightarrow 0$ in $H_{0}^{1}(\Omega), w_{n} \rightarrow 0$ strongly in $L^{2}(\Omega)$ and this is a contradiction with $\left\|w_{n}\right\|_{L^{2}(\Omega)}=1$. This ends the proof. 
Proof of Corollary 1.6. Let us consider a simple eigenvalue $\lambda$ and $e$ be denoted the associated eigenfunction. By Theorem 1.5 we had to show that the function

$$
J(\Gamma)=\Gamma^{2} \int_{\Omega} e^{2}(2 \log |\Gamma e|-1) s
$$

admits only one pairs of critical points $\pm \gamma$ verifying $J^{\prime \prime}( \pm \gamma) \neq 0$. A straightforward computation shows that

$$
J^{\prime}(\gamma)=0 \Leftrightarrow \gamma= \pm \exp \left(-\int_{\Omega} e^{2} \log |e|\right) .
$$

Corresponding to these values of $\gamma$ we get

$$
\begin{aligned}
J^{\prime \prime}( \pm \gamma) & =2 \int_{\Omega}\left(e^{2} \log |\gamma e|+e^{2}\right)=2 \log |\gamma|+2 \int_{\Omega} e^{2} \log |e|+2 \\
& =2 \neq 0 .
\end{aligned}
$$

which proves the claim.

\section{References}

[1] D. Bonheure, V. Bouchez, C. Grumiau and J. Van Schaftingen, Asymptotics and symmetries of least energy nodal solutions of Lane-Emden problems with slow growth, Commun. Contemp. Math. 10 (2008), 1-23.

[2] D. C. Clark, A variant of the Lusternik-Schnirelman theory, Indiana Univ. Math. J. 22 (1972/73), 65-74.

[3] E. N. DANCER, Real analyticity and non-degeneracy, Math. Ann. 325 (2003), 369-392.

[4] E. N. DANCER, Y. DU and L. MA, Asymptotic behavior of positive solutions of some elliptic problems, Pacific J. Math. 210 (2003), 215-228.

[5] C. S. LIN, Uniqueness of least energy solutions to a semilinear elliptic equation in $R^{2}$, Manuscripta Math. 84 (1994), 13-19.

[6] A. M. Micheletti, Perturbazione dello spettro dell'operatore di Laplace, in relazione ad una variazione del campo, Ann. Scuola Norm. Sup. Pisa Cl. Sci. 26 (1972), 151-169.

[7] P. H. Rabinowitz, "Minimax Methods in Critical Point Theory with Applications to Differential Equations", CBMS Regional Conference Series in Mathematics, Amer. Math. Soc., Vol. 65, Providence RI, 1986.

[8] K. UHLENBECK, Generic properties of eigenfunctions, Amer. J. Math. 98 (1976), 1059-1078.

Dipartimento di Matematica

Sapienza Università di Roma Piazzale A. Moro, 2 00185 Roma, Italia grossi@mat.uniroma1.it 